Public Abstract

First Name:Christopher

Middle Name:James

Last Name:Melick

Adviser's First Name:Patrick

Adviser's Last Name:Market

Co-Adviser's First Name:

Co-Adviser's Last Name:

Graduation Term:SP 2008

Department:Soil \& Atmospheric Science

Degree:PhD

\title{
Title:ON THE SYNOPTIC AND MESOSCALE ORGANIZATION OF MID-LATITUDE, CONTINENTAL CONVECTIVE SNOW EVENTS
}

An ingredients-based methodology was pursued in order to evaluate the likelihood of thunderstorms occurring in the presence of snowfall (i.e. thundersnow; TSSN). In order to properly distinguish from typical snowstorms (i.e. non-TSSN), the detailed examination focused on stability characteristics of wintertime convection across the central United States immediately leading up to the onset of the event. More specifically, the research primarily analyzed the value of the seldom applied growth rate parameter. Identification of a separate collection of non-TSSN events helped to highlight the differences, and ultimately, the significance in the findings for the TSSN subset.

The current work substantiated the premise that atmospheres were more unstable in episodes of convective snow with the analyses also revealing pronounced forcing mechanisms. The development of TSSN and any associated banding was correctly and most accurately predicted from trends in plots of growth rates analyzed at the level at which the highest significant growth rates occurred. An outlook can be more accurately issued by identifying regions where reduced values of equivalent potential vorticity (i.e. small symmetric stability or instability) are collocated with estimates of high growth rates (i.e. where smallscale slantwise perturbations will grow). Given the overall success, it is hoped that some of the conclusions established by this work will be implemented routinely in an operational environment and provide forecasters an additional, essential tool in dealing with nowcasting situations of hazardous winter weather events. 